\title{
Métodos de Elementos Finitos de Arestas para as Equações de Maxwell 3D
}

\author{
Renan Henrique Luz Seluzniak Saulo Pomponet Oliveira \\ Departamento de Matemática, Universidade Federal do Paraná, \\ 81531-980, Curitiba, PR \\ E-mail: renan_seluzniak@yahoo.com.br, saulopo@ufpr.br.
}

\section{RESUMO}

Este trabalho trata da aproximação do seguinte problema de valores de contorno:

$$
\left\{\begin{array}{l}
\nabla \times\left(\mu^{-1} \nabla \times \vec{E}\right)-\omega^{2} \tilde{\epsilon} \vec{E}=\vec{F}, \quad x \in \Omega, \\
\vec{E} \times \vec{n}=\overrightarrow{0}, \quad x \in \Gamma,
\end{array}\right.
$$

dado pelas equações de Maxwell de segunda ordem no regime harmônico considerando a frequência $\omega$ e condições de contorno para um condutor perfeito [1,2]. Neste problema, $\tilde{\epsilon}=\epsilon+i \sigma / \omega$, em que $\sigma, \mu$ e $\epsilon$ representam a condutividade elétrica, a permeabilidade magnética e a permissividade elétrica, respectivamente. Considerou-se que o domínio $\Omega$ é tridimensional.

A principal contribuição deste trabalho é generalizar para três dimensões o trabalho desenvolvido em [3], no qual considera-se a aproximação do problema (1) pelo método de elementos finitos de arestas de ordem zero. Esta variante do método de elementos finitos evita soluções espúrias no regime de baixas frequências, conforme ocorre com a versão vetorial do método de elementos finitos tradicional com funções nodais [2].

Nesta implementação foi considerado um domínio cúbico particionado em elementos finitos cúbicos.

A Figura 1 ilustra a numeração dos vértices e elementos realizada no caso particular uma malha de $2 \times 2 \times 2$ cubos, além da numeração das arestas no elemento de referência $[-1,1] \times[-1,1] \times[-1,1]$.

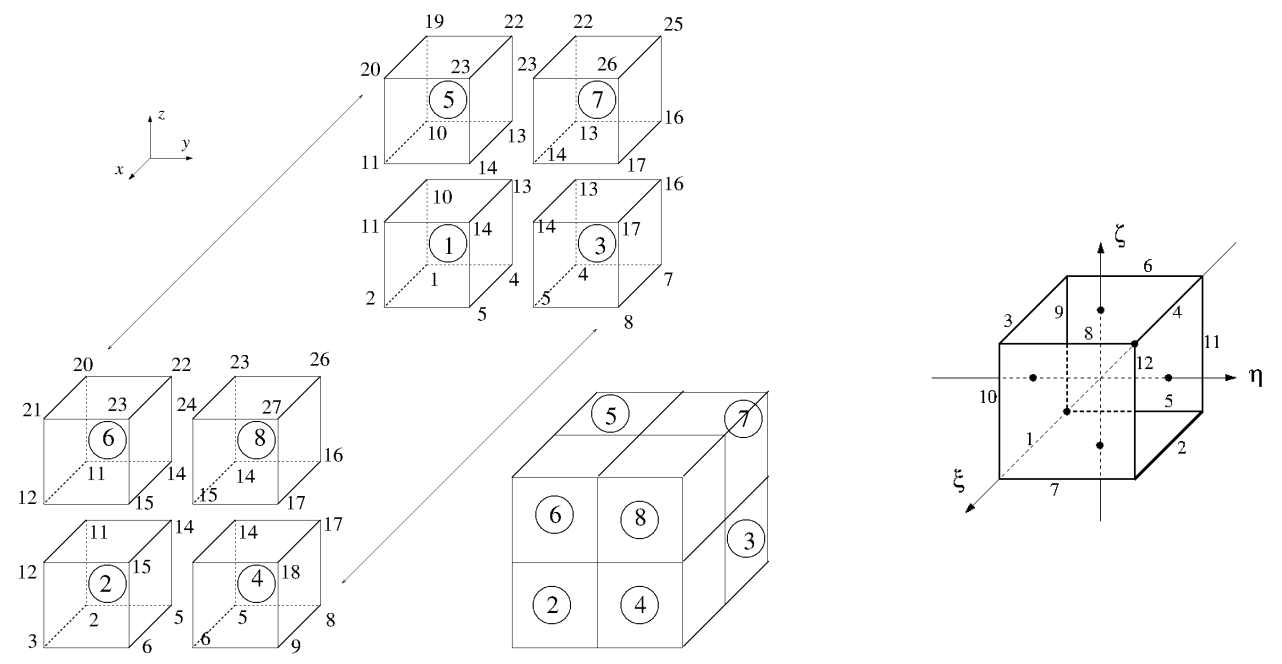

Figura 1: O sentido da contagem dos vértices, elementos e arestas. 
As funções de base de ordem zero no elemento de referência são dadas por:

$$
\begin{array}{ll}
\hat{\phi}_{1}(\xi, \eta, \zeta)=[(1-\eta)(1-\zeta) / 4,0,0]^{T}, & \hat{\phi}_{2}(\xi, \eta, \zeta)=[(1+\eta)(1-\zeta) / 4,0,0]^{T}, \\
\hat{\phi}_{3}(\xi, \eta, \zeta)=[(1-\eta)(1+\zeta) / 4,0,0]^{T}, & \hat{\phi}_{4}(\xi, \eta, \zeta)=[(1+\eta)(1+\zeta) / 4,0,0]^{T}, \\
\hat{\phi}_{5}(\xi, \eta, \zeta)=[0,(1-\xi)(1-\zeta) / 4,0]^{T}, & \hat{\phi}_{6}(\xi, \eta, \zeta)=[0,(1-\xi)(1+\zeta) / 4,0]^{T}, \\
\hat{\phi}_{7}(\xi, \eta, \zeta)=[0,(1+\xi)(1-\zeta) / 4,0]^{T}, & \hat{\phi}_{8}(\xi, \eta, \zeta)=[0,(1+\xi)(1+\zeta) / 4,0]^{T}, \\
\hat{\phi}_{9}(\xi, \eta, \zeta)=[0,0,(1-\xi)(1-\eta) / 4]^{T}, & \hat{\phi}_{10}(\xi, \eta, \zeta)=[0,0,(1+\xi)(1-\eta) / 4]^{T}, \\
\hat{\phi}_{11}(\xi, \eta, \zeta)=[0,0,(1-\xi)(1+\eta) / 4]^{T}, & \hat{\phi}_{12}(\xi, \eta, \zeta)=[0,0,(1+\xi)(1+\eta) / 4]^{T} .
\end{array}
$$

Foi validado esta implementação com um experimento numérico em que foi comparado a solução obtida pelo algoritmo com uma solução particular do problema (1). Esta solução é obtida escolhendo-se a fonte $\vec{F}$ de modo que o campo vetorial $\vec{E}$ seja dado por

$$
\vec{E}=[\operatorname{sen}(M \pi y) \operatorname{sen}(M \pi z), \operatorname{sen}(M \pi x) \operatorname{sen}(M \pi z), \operatorname{sen}(M \pi x) \operatorname{sen}(M \pi y)]^{T} .
$$

No caso particular em que $\mu=1, \quad \epsilon=1, \quad \sigma=0$, após algumas manipulações algébricas obtevese:

$$
\vec{F}=\left[\begin{array}{l}
\left(2 M^{2} \pi^{2}-\omega^{2} \tilde{\epsilon}\right) \operatorname{sen}(M \pi y) \operatorname{sen}(M \pi z) \\
\left(2 M^{2} \pi^{2}-\omega^{2} \tilde{\epsilon}\right) \operatorname{sen}(M \pi x) \operatorname{sen}(M \pi z) \\
\left(2 M^{2} \pi^{2}-\omega^{2} \tilde{\epsilon}\right) \operatorname{sen}(M \pi x) \operatorname{sen}(M \pi y)
\end{array}\right]
$$

A Figura 2 compara a solução obtida pelo método de elementos finitos de arestas e a solução exata do problema para uma malha de $20 \times 20 \times 20$ cubos. Neste experimento, utilizou-se $M=3$.
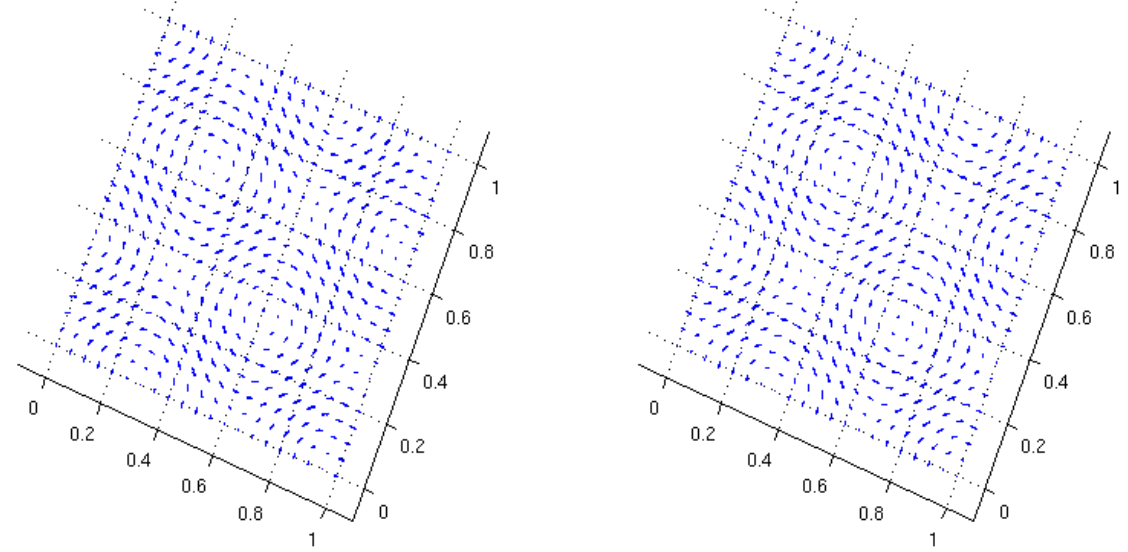

Figura 2: Corte, no plano $x y$, da solução do algoritmo (à esquerda) e a solução exata (à direita).

Para trabalhos futuros, o uso de métodos iterativos para sistemas lineares poderia permitir o uso do programa com malhas com um maior número de pontos. Outro interessante desenvolvimento futuro é a adaptação do programa para malhas não necessariamente formadas por elementos cúbicos.

Palavras-chave: Elementos finitos de arestas, Equações de Maxwell no regime harmônico, elementos de Whitney

\section{Referências}

[1] J. Jin, "The Finite Element Method in Electromagnetism", 2nd ed. New York: John Wiley and Sons, 2002.

[2] P. Monk, "Finite Element Methods for Maxwell's Equations", New York: Oxford University Press, 2003.

[3] J.E. Sebold, "Métodos de Elementos Finitos de Nédélec para as Equações de Maxwell Harmônicas no Tempo", Dissertação de Mestrado, Universidade Federal do Paraná, 2011. 